\title{
Role of C1q-binding anti-HLA antibodies as a predictor of lung allograft outcome
}

\author{
Olivier Brugière ${ }^{1}$, Antoine Roux ${ }^{2,8}$, Jerome Le Pavec ${ }^{3,8}$, Deborah Sroussi ${ }^{1}$, \\ François Parquin ${ }^{2}$, Pauline Pradère ${ }^{3}$, Clairelyne Dupin ${ }^{1}$, Vincent Bunel (1) ${ }^{1}$, \\ Gisele Mourin ${ }^{1}$, Gilles Jebrak ${ }^{1}$, Gaëlle Dauriat ${ }^{1}$, Yves Castier ${ }^{4}$, Pierre Mordant ${ }^{4}$, \\ Brice Lortat-Jacob ${ }^{5}$, Sylvain Jean-Baptiste $\mathbb{1}^{5}$, Herve Mal ${ }^{1}$, \\ Caroline Suberbielle ${ }^{6}$, Chantal Gautreau ${ }^{6}$, Sophie Caillat-Zucman ${ }^{6}$, \\ Aurélie Cazes ${ }^{7}$, Gabriel Thabut ${ }^{1,9}$ and Jean-Luc Taupin ${ }^{6,9}$
}

Affiliations: 'Service de Transplantation Pulmonaire, Bichat Hospital, Paris, France. ${ }^{2}$ Service de Transplantation Pulmonaire, Foch Hospital, Suresnes, France. ${ }^{3}$ Service de Transplantation Pulmonaire, Centre Chirugical Marie Lannelongue, Le Plessis Robinson, France. ${ }^{4}$ Service de Chirurgie Thoracique, Bichat Hospital, Paris, France. ${ }^{5}$ Service de Réanimation Chirurgicale, Bichat Hospital, Paris, France. ${ }^{6}$ Laboratoire d'Immunologie et Histocompatibilité, Saint-Louis Hospital, Paris, France. ${ }^{7}$ Service d'Anatomo-Pathologie, Bichat Hospital, Paris, France. ${ }^{8}$ These authors contributed equally to the study. ${ }^{9}$ These authors contributed equally to the study.

Correspondence: Olivier Brugière, Service de Pneumologie B et Transplantation Pulmonaire, Hôpital Bichat, 46 rue Henri Huchard, 75018 Paris, France. E-mail: olivier.brugierelabch.aphp.fr

@ERSpublications

The Clq assay allows for a better identification of lung-transplant recipients with the highest risk of graft failure http://ow.ly/CkmY30kz7P8

Cite this article as: Brugière $\mathrm{O}$, Roux A, Le Pavec J, et al. Role of C1q-binding anti-HLA antibodies as a predictor of lung allograft outcome. Eur Respir J 2018; 52: 1701898 [https://doi.org/10.1183/ 13993003.01898-2017].

ABSTRACT Presence of anti-human leukocyte antigen donor-specific antibodies (DSAs) is associated with poor outcome after lung transplantation. Currently, DSAs are detected using the Luminex technique, which may be overly sensitive. The new $\mathrm{Clq}$ assay allows for the exclusive detection of complement (C1q)-binding antibodies, involved in antibody-mediated rejection. We investigated whether early detection of complement-binding DSAs is associated with chronic lung allograft dysfunction (CLAD) and survival.

From 2009 to 2012, lung transplant recipients from three transplantation centres were screened for the presence of DSA and their complement-binding capacity during the 6-12 months post-transplantation in a stable condition.

The analysis included 168 patients. The 3-year rates of freedom from CLAD and graft survival were lower for patients with complement-binding DSAs (33.6\% and 53.7\%, respectively), as compared with patients with non-complement-binding DSAs (61.9\% and $77.4 \%$, respectively) and patients without DSA (70\% and $84.9 \%$, respectively) ( $\mathrm{p}<0.001$ and $\mathrm{p}=0.001$, respectively). Detection of complement-binding DSA was associated with a risk of graft loss that was nearly tripled after adjustment for clinical, functional, histological and immunological factors (hazard ratio 2.98, 95\% CI 1.33-6.66; $\mathrm{p}=0.008$ ).

Assessment of the C1q-binding capacity of DSA appears to be useful in identifying stable lung transplant recipients at high risk of lung allograft loss. 


\section{Introduction}

The potential destructive role of alloantibodies directed against donor human leukocyte antigen (HLA) in survival was first documented more than four decades ago after renal transplantation [1]. In lung transplantation, there is evidence for the deleterious role of donor-specific antibodies (DSAs) in the development of chronic lung allograft dysfunction (CLAD) and graft loss [2-12], mostly after the introduction of solid phase assays: first ELISA, then bead-based technology and then Luminex-based assays including single-antigen flow bead (SAFB) in the early 2000s [13]. Since the implementation of Luminex technology with increased sensitivity in most transplantation centres, the proportion of lung transplantation recipients with detected anti-HLA antibodies has increased dramatically, reaching about half of recipients in some centres $[9,14]$. Nevertheless, critics consider SAFB assay to be overly sensitive, and how to interpret and apply these results clinically continues to be debated [15], especially for Luminex-detected DSAs in stable lung transplantation recipients. Since the discovery that anti-HLA antibodies are lymphocytotoxic, the activation of the complement cascade has been considered a key component in antibody-mediated rejection (AMR), and C4d deposition in capillaries has been considered the footprint of AMR damage in kidney transplantation [16-18] and more recently in lung transplantation [19-21]. The capacity of anti-HLA antibodies to bind complement fraction C1q, the first step in activating the classical complement cascade, determines their cytotoxic potential [18]. Similarly, it is hypothesised in lung transplantation that only some of these Luminex-detected DSA antibodies with the ability to fix the complement may be involved in lung graft injury of AMR, whereas non-complement-fixing antibodies are considered less or non-deleterious. These uncertainties of precise markers of humoral rejection are reflected by the recent consensus report of the International Society for Heart and Lung Transplantation (ISHLT) on lung AMR [19], which defined the degree of certainty of AMR diagnosis depending on the demonstration of presence or absence of multiple criteria (allograft dysfunction, lung histology, C4d staining and presence of DSAs). Hence, although DSA antibodies are considered harmful, the spectrum of graft injury related to these antibodies ranges widely, from no recognised damage to graft loss [12, 22, 23].

A novel $\mathrm{C} 1 \mathrm{q}$ assay has been developed to define the subset of antibodies likely to be clinically significant, but having the sensitivity of the SAFB assay [24]. We hypothesised that the complement-binding properties of DSA antibodies are involved in CLAD onset and lung graft failure, and could better stratify the immunological risk after lung transplantation, in an attempt to improve the identification of patients requiring desensitisation treatment. Hence, we conducted a multicentre study to determine whether assessing the presence of C1q-binding DSA antibodies in the first year after lung transplantation might improve risk stratification for CLAD occurrence and lung allograft loss.

\section{Patients and methods}

We reviewed data for all consecutive patients who underwent lung transplantation between January 2009 and December 2012 at the hospitals Bichat, Foch and Marie-Lannelongue (France), and who survived $\geqslant 6$ months after transplantation. All patients with at least one available anti-HLA antibodies detection performed using SAFB Luminex assay (One Lambda, Canoga Park, CA, USA) between 6 and 12 months after transplantation and who were free of CLAD at the date of Luminex SAFB testing were included in this study. The HLA typing and crossmatching for this cohort have been published previously [25], and are detailed in the online supplementary material. HLA typing of recipients was performed using molecular biology (Innolipa HLA typing kit; Innogenetics, Ghent, Belgium). For all lung transplant donors, HLA-A/B/DR/DQ tissue typing involved the microlymphocytotoxicity technique with tissue-typing trays (One Lambda), and was controlled using molecular biology.

\section{Follow-up}

The monitoring protocols for surveillance of acute rejection episodes, pulmonary infections and chronic allograft dysfunction (CLAD) during follow-up used at Bichat hospital have been described previously [26, 27] and those of Foch and Marie-Lannelongue hospital are detailed in the online supplementary material. CLAD were classified into two clinical phenotypes: bronchiolitis obliterans syndrome (BOS), defined by the classical ISHLT definition [28] or restrictive allograft syndrome (RAS) [29, 30]. Severe RAS was defined as requiring oxygen therapy.

\section{Immunosuppression}

The protocol of immunosuppression used at Bichat hospital has been described previously [26], and those of Foch and Marie-Lannelongue hospitals are detailed in the online supplementary material. This study was approved by an institutional ethics committee, and conducted in accordance with good clinical practices and the recommendations concerning human research contained in the Declaration of Helsinki. 
Detection and characterisation of DSAs

Historical Luminex SAFB assay

From January 2009, routine Luminex assay was implemented in the laboratory of Saint-Louis hospital for screening of anti-HLA antibodies directed against HLA class I and class II antigens (LSA; One Lambda), and identification by SAFB Luminex assay, as previously reported and detailed in the online supplementary material. Patients were screened by Luminex assay at day 0, month 1 and then every 3 months in the first postoperative year, and additionally at the discretion of the physician in the three centres. All beads with a normalised mean fluorescence intensity (MFI) $>500$ were considered positive, and the DSA nature of the detected antibody was assigned when at least one bead bearing the donor antigen was above the positivity threshold. In case of DSA detected by SAFB Luminex assay within the first 6-12 months after transplantation, the serum with the highest value of MFI for the immunodominant DSA and associated with a concomitant functional stability (absence of CLAD) was selected. For all patients included in this study, all sera screened by SAFB Luminex assay within 6-12 months post-transplantation were reviewed, with a mean number of DSA screenings within the 6-12 months after transplantation of 2.6 \pm 1.9 SAFB assays per patient. With absence of DSAs in any available serum test with Luminex SAFB assay within the 6-12 months after transplantation, we choose the serum sample closest to the 6-month post-transplantation date. Only one serum sample was selected per patient. The choice of the 6-12-month period post-lung transplantation to assess the presence of DSA was made according to previous studies which reported a median time to the development of DSA between 6 and 12 months post-lung transplantation $[5,6,31]$.

\section{EDTA-Luminex assay}

For all included patients with DSAs detected within 6-12 months after transplantation, the selected historical serum sample was additionally retested for identification of circulating DSA antibodies with a SAFB Luminex assays modified using EDTA pre-treatment of serum. DSAs identified by SAFB Luminex assay with EDTA were also reinterpreted and named reinterpreted EDTA-DSA (r-DSA) (online supplementary material).

\section{C1q assay}

For all patients, each historical selected serum sample with detection of DSA or r-DSA was then retested with use of SAFB Luminex C1q assay in a blinded fashion at the immunology laboratory of Saint-Louis Hospital by technicians who were unaware of the patient's status. The assay involved the C1q-Screen kit (One Lambda), according to the manufacturer's recommendations (online supplementary material).

\section{Statistical analysis}

Continuous variables are presented as mean $\pm \mathrm{SD}$ or median (range), and were compared using t-test or Mann-Whitney U-test. Categorical variables are presented as $\mathrm{n}(\%)$ and were compared using Chi-squared test or Fisher's exact test. Correlations between continuous variables were assessed by Spearman's rank correlation coefficient.

Time to CLAD onset (freedom from CLAD) and time to death or retransplantation (graft survival) were estimated using the Kaplan-Meier estimator and compared by log-rank test. Graft survival, freedom from $\mathrm{CLAD}$ and follow-up were computed from the time of $\mathrm{Clq}$ testing in all patients. Univariate and multivariable Cox models were built to assess the association between the presence of complement-fixing or non-fixing DSAs and freedom from CLAD survival and graft survival with adjustment for potential confounding factors. Factors associated with freedom from CLAD and graft survival by univariate analyses (at a significance of $\mathrm{p}<0.2$ ) were selected for multivariate analyses. DSA status (positive or negative) was assessed between 6 and 12 months after lung transplantation, and was thus included in the Cox model as a time independent covariate. Follow-up was assessed from the date of $\mathrm{Clq}$ testing for all patients. The last follow-up date for all survival models was June 2016. The centre effect was accounted for by allowing different baseline hazard functions in each centre in the Cox models (strata option: coxph function in R). The proportional hazards assumption was tested using Grambsch and T.M. Therneau tests based on weighted residuals (cox.zph function in R) [32]. Quantification of follow-up was performed using the Kaplan-Meier estimate of potential follow-up [33]. For all analyses, $\mathrm{p}<0.05$ was considered statistically significant. Analyses used Stata v12 for Macintosh (StataCorp, College Station, TX, USA). This study was approved by an institutional ethics committee, and conducted in accordance with good clinical practices and the recommendations concerning human research contained in the Declaration of Helsinki.

\section{Results}

\section{Patient characteristics}

We included 168 patients (70 at Bichat, 58 at Foch and 40 at Marie-Lannelongue hospital) in the main analysis. Patients were classified into three groups, as follows. 1) Patients without DSA antibodies $(\mathrm{n}=100)$; 
2) patients with non-complement-binding DSAs $(n=41)$; and 3) patients with complement-binding DSAs $(\mathrm{n}=27)$ (figure 1). Table 1 describes the baseline characteristics of the cohort according to historical DSA classification. Frequency of DSA detection was higher for male than female recipients $(p=0.02)$. We compared the immunosuppressive therapy between patients with $\mathrm{C}_{1 \mathrm{q}^{+}} \mathrm{DSA}^{+}$with the other patients (those with no DSA or C1q-DSAs) and found no difference between groups with regard to the presence of an induction therapy (with thymoglobulin or interleukin-2 receptor antibodies) $(p=0.62)$ and percentage of patients receiving tacrolimus versus cyclosporine $(\mathrm{p}=0.98)$. Frequency of preformed DSAs and HLA-DR and HLA-DQ donor-recipient mismatches (MM) were higher with $\mathrm{DSA}^{+} \mathrm{Clq}^{-}$and $\mathrm{DSA}^{+} \mathrm{Clq}^{+}$patients than those without DSA (preformed DSA $44 \%$ and $44 \%$ versus $29 \%, \mathrm{p}=0.03$; HLA-DR MM $63 \%$ and $72 \%$ versus $42 \%, \mathrm{p}=0.004$; HLA-DQ MM $41 \%$ and $47 \%$ versus $35 \%, \mathrm{p}=0.03$ ). Other patient characteristics did not differ between the three patient groups.

\section{Freedom from CLAD and graft survival according to detection of DSA and complement-binding properties \\ Freedom from CLAD}

The median (95\% CI) follow-up after C1q assessment was 4.2 (4.0-4.5) years and did not significantly differ among the three patient groups (4.3 (4.0-4.5) years, $4.0(3.8-4.8)$ years and $4.6(4.0-\infty)$ years, respectively; $\mathrm{p}=0.28)$. CLAD developed in $72(42.9 \%)$ patients during follow-up. One patient died within 1 month following the date of C1q sampling, but did not develop CLAD before death and was simply censored for assessment of freedom from CLAD. Freedom from CLAD was lower in patients with DSAs $(\mathrm{n}=68)$ than in patients without DSA $(\mathrm{n}=100)$ : 1 - and 3-year freedom frequencies $(95 \% \mathrm{CI})$ were $71 \%$ $(61.8-83.4 \%)$ and $51.0 \%(40.1-64.8 \%)$ versus $86.7 \%(80.2-93.7 \%)$ and $70.6 \%(62.0-80.4 \%)$ (hazard ratio (HR) 2.1, 95\% CI 1.3-3.3; p=0.002) (figure 2a). When patients with DSAs after transplantation were subsequently categorised according to complement-binding capacity, patients with C1q-binding DSAs had the poorest 1-year and 3-year rates of freedom from CLAD (54.3\% (38.2-77.2\%) and 33.6\% (19.4-58.4\%), respectively; HR 3.36, 95\% CI 1.9-5.9), as compared with patients with non-C1q-binding DSAs (82.9\% (72.2-95.3\%) and 61.9\% (48.3-79.3\%), respectively; HR 1.49, 95\% CI 0.85-2.62) and patients without DSA (86.7\% (80.2-93.7\%) and 70.6\% (62.0-80.4\%), reference group) $(\mathrm{p}<0.0001)$ (figure $2 \mathrm{~b})$.

\section{Graft survival}

Overall, 43 (25.6\%) patients died, 10 (6.0\%) underwent retransplantation and 115 (68.5\%) were still alive at the end of follow-up. Graft survival frequency was lower in patients with than without DSAs: the 1- and 3-year graft survival frequencies (95\% CI) were $88.2 \%(80.9-96.2 \%)$ and $68.1 \%(57.7-80.4 \%)$ versus $95.0 \%$ (90.8-99.4\%) and 84.9\% (78.2-92.3\%) (HR 2.0, 95\% CI 1.2-3.4; $\mathrm{p}=0.01$ ) (figure 3a). When patients with DSAs after transplantation were subsequently categorised according to complement-binding capacity, patients with C1q-binding DSAs had the poorest 1-year and 3-year graft survival (81.5\% (68.1-97.5\%) and $53.7 \%$ (37.5-77.1\%); HR 3.1, 95\% CI 1.6-5.9), as compared with those with non-C1q-binding DSA (92.7\%

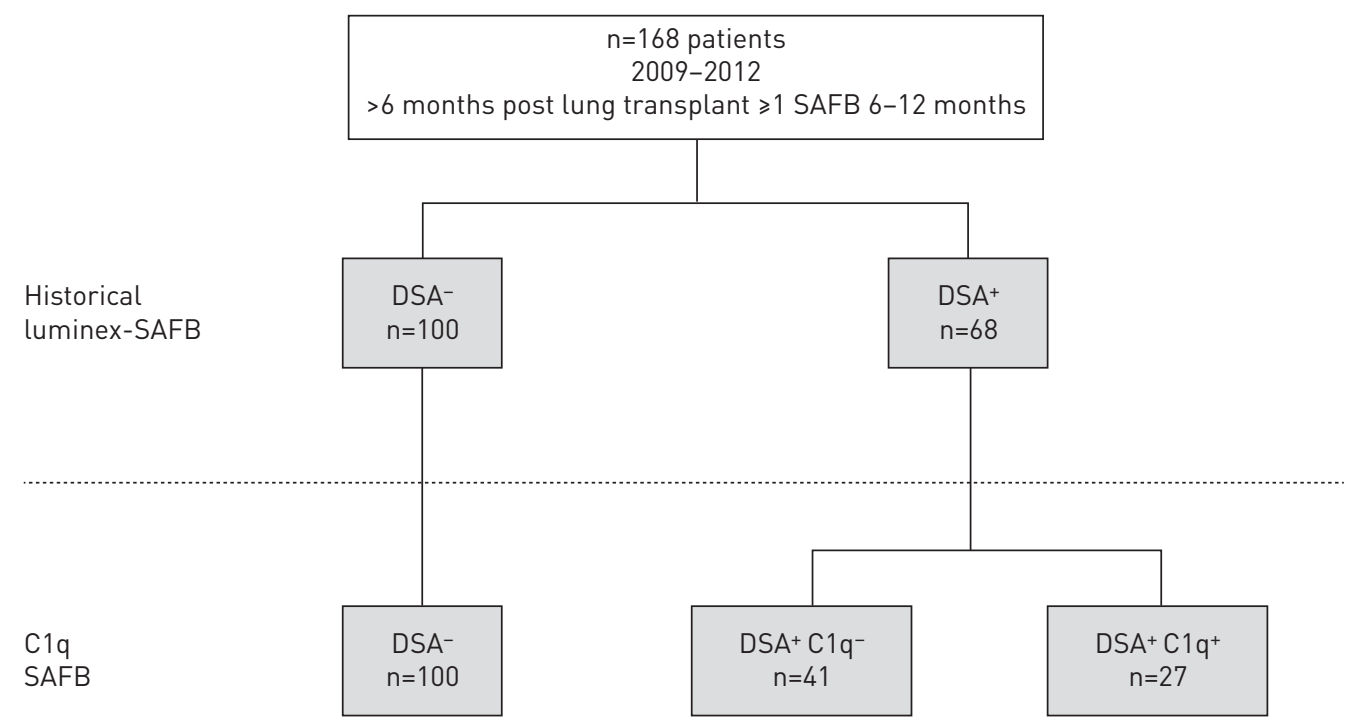

FIGURE 1 Study flow chart according the detection of historical single-antigen flow bead (SAFB)/C1q. DSA: donor-specific anti-human leukocyte antigen antibody. 
TABLE 1 Baseline characteristics of the study population by presence or absence of donor-specific anti-human leukocyte antigen (HLA) antibodies (DSAs) without or with C1q binding after lung transplantation

\begin{tabular}{|c|c|c|c|c|c|}
\hline & \multirow[t]{2}{*}{ All patients } & \multirow[t]{2}{*}{ Patients without DSA } & \multicolumn{2}{|c|}{ Patients with DSA } & \multirow[t]{2}{*}{$p$-value $e^{\pi,+}$} \\
\hline & & & $\mathrm{DSA}^{+} \mathrm{C} \mathrm{q}^{-}$ & $\mathrm{DSA}^{+} \mathrm{C} 1 \mathrm{q}^{+\#}$ & \\
\hline Patients & 168 & 100 & 41 & 27 & \\
\hline \multicolumn{6}{|l|}{ Recipients } \\
\hline Age years & $44.8 \pm 14.2$ & $45.7 \pm 13.9$ & $43.6 \pm 14.0$ & $40.2 \pm 16.8$ & 0.14 \\
\hline Female & $91(54)$ & $61(61)$ & $21(51)$ & 9 (33) & 0.02 \\
\hline Sex mismatch & $41(24)$ & $24(24)$ & $11(27)$ & $6(22)$ & 0.75 \\
\hline Diagnosis & & & & & 0.29 \\
\hline COPD or $\alpha_{1}-\mathrm{AT}$ & $42(25)$ & $31(31)$ & $8(19)$ & $3(11)$ & \\
\hline Cystic fibrosis & $47(28)$ & $22(22)$ & $13(31)$ & $12(44)$ & \\
\hline Fibrosis & $40(24)$ & $28(28)$ & $9(22)$ & $3(11)$ & \\
\hline PAH & $29(17)$ & $14(14)$ & $9(22)$ & $6(22)$ & \\
\hline Other & $9(5)$ & $5(5)$ & $2(4)$ & $3(11)$ & \\
\hline Type of lung transplant & & & & & 0.57 \\
\hline Bilateral & $97(58)$ & $61(61)$ & $21(51)$ & $15(55)$ & \\
\hline $\mathrm{CP}$ & $15(8)$ & $7(7)$ & $4(10)$ & $4(15)$ & \\
\hline Unilateral & $56(33)$ & 32 (32) & $16(39)$ & $8(30)$ & \\
\hline \multicolumn{6}{|l|}{ Baseline lung function ${ }^{\S}$} \\
\hline $\mathrm{FEV} 1 \mathrm{~mL}$ & & $2012 \pm 74$ & $2116 \pm 117$ & $2363 \pm 180$ & 0.13 \\
\hline FEV $1 \%$ pred & & $67.1 \pm 2.0$ & $68.4 \pm 3.3$ & $69.3 \pm 3.4$ & 0.87 \\
\hline CMV mismatch $\left(D^{+} / R^{-}\right)$ & $31(18)$ & $18(18)$ & $8(19)$ & $5(18)$ & 0.98 \\
\hline Per-operative ECMO & $69(41)$ & $38(38)$ & $17(41)$ & $14(52)$ & 0.38 \\
\hline PGD grade 3 versus $0 / 1 / 2$ & $39(23)$ & $24(24)$ & $10(24)$ & $5(18)$ & 0.86 \\
\hline $\begin{array}{l}\text { Ischaemic time min } \\
\text { Immunological characteristics }\end{array}$ & $305 \pm 91$ & $310 \pm 78$ & $306.5 \pm 81.3$ & $309 \pm 98$ & 0.94 \\
\hline HLA A MM (2 versus 01) & $89(53)$ & $52(53)$ & $27(65)$ & $10(37)$ & 0.09 \\
\hline HLA B MM (2 versus 01) & $124(74)$ & $75(76)$ & $30(73)$ & $19(70)$ & 0.91 \\
\hline HLA DR MM (2 versus 01) & $87(51)$ & $43(43)$ & $26(63)$ & $18(66)$ & 0.004 \\
\hline HLA DQ MM (2 versus 01) & $61(36)$ & $31(31)$ & $19(46)$ & $11(40)$ & 0.03 \\
\hline Class I DSA & & & $9(21)$ & $2(7)$ & \\
\hline A & & & $4(10)$ & $2(7)$ & \\
\hline $\mathrm{B}$ & & & $3(7)$ & $0(0)$ & \\
\hline C & & & $2(5)$ & $0(0)$ & \\
\hline Class II DSA & & & $32(78)$ & 25 (92) & \\
\hline DR & & & $8(19)$ & $4(15)$ & \\
\hline $\mathrm{DQ}$ & & & $22(53)$ & $19(70)$ & \\
\hline DP & & & 2 (5) & $2(7)$ & \\
\hline Crossmatch-positive $^{f}$ & & $4(8)$ & $3(12)$ & $2(10)$ & 0.94 \\
\hline Preformed DSA & $37(31)$ & $16(22)$ & $13(44)$ & $8(44)$ & 0.03 \\
\hline
\end{tabular}

Data are presented as $\mathrm{n}$, mean \pm SD or $\mathrm{n}(\%)$, unless otherwise stated. COPD: chronic obstructive pulmonary disease; $\alpha_{1}$-AT: $\alpha_{1}$-antitrypsin; PAH: pulmonary arterial hypertension; CP: cardiopulmonary; FEV 1 : forced expiratory volume in $1 \mathrm{~s}$; CMV: cytomegalovirus; ECMO: extra-corporeal membrane oxygenation; PGD: primary lung graft dysfunction; $M M$ : mismatch. \#: $p$-values are for the comparison between nonC1q-binding donor-specific anti-HLA antibodies and C1q-binding donor-specific anti-HLA antibodies; П: $p<0.05$ for the comparison between no donor-specific anti-HLA antibodies and presence of DSAs; ${ }^{+}$: Chi-squared tests were used for the comparison of categorical variables, and the unpaired t-test for the comparison of continuous variables; ${ }^{\S}$ : data are presented as mean \pm SEM; ${ }^{f}$ : in patients with available crossmatch $\mathrm{T}$ or $\mathrm{B}$

(85.0-100\%) and 77.4\% (65.3-91.6\%); HR 1.4, 95\% CI 0.7-2.8) and without DSA (95.0\% (90.8-99.4\%) and $84.9 \%(78.2-92.3 \%)$, reference group) $(\mathrm{p}=0.001)$ (figure $3 \mathrm{~b}$ ).

Freedom from CLAD and graft survival according to reinterpreted r-DSA and complement-binding properties

In addition, we analysed freedom from CLAD and graft survival according to reinterpreted Luminex EDTA-SAFB DSAs (r-DSAs) status after transplantation, and observed similar results to those with historical DSAs (online supplementary figure S1). Patients with C1q-binding r-DSAs had the poorest 1-year and 3-year freedom from CLAD $(34.2 \%(15.5-75.2 \%)$ and $17.1 \%(4.9-60.1 \%)$, respectively) and the poorest 1-year and 3-year graft survival (76.9\% (57.1-100\%) and 38.5\% (19.3-76.5\%), respectively). Details 

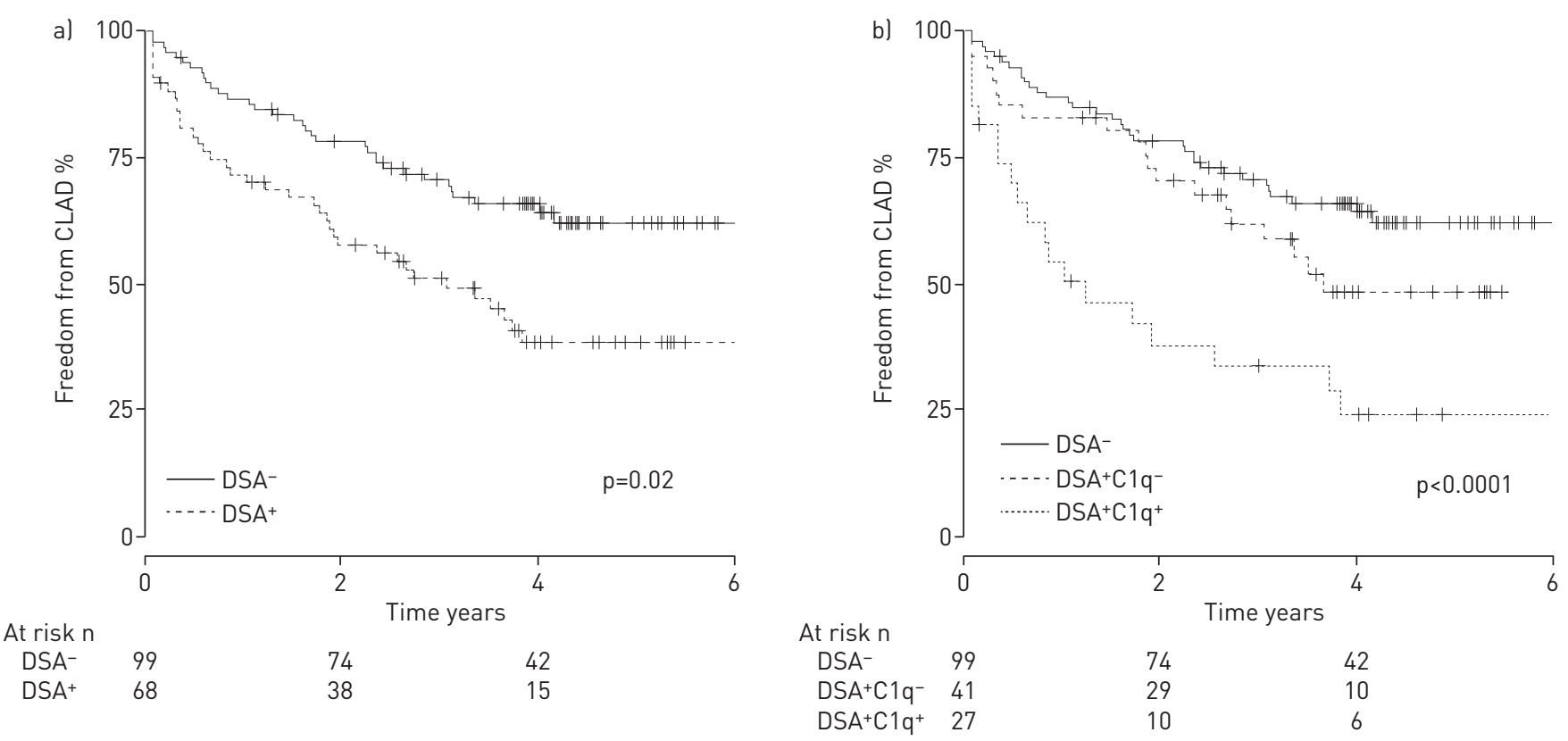

FIGURE 2 Kaplan-Meier curves for freedom from chronic allograft dysfunction (CLAD) according to donor-specific anti-human leukocyte antigen antibody (DSA) status after transplantation. Probability of freedom from CLAD a) by presence or absence of DSA detected by classical historical Luminex single-antigen flow bead assay; b) by presence or absence of DSAs and their C1q-binding capacity. Survival curves are marked by a vertical tick at each censoring time

of analysis of CLAD and graft survival according to r-DSAs and their complement-binding properties are detailed in online supplementary figures S2 and S3.

Determinants of CLAD onset and graft failure

The association of clinical, functional, histological and immunological factors with CLAD onset and graft loss were assessed in univariate analysis and backward-elimination multivariate Cox regression analyses. Several variables showed association with CLAD onset and graft survival in univariate analysis, including
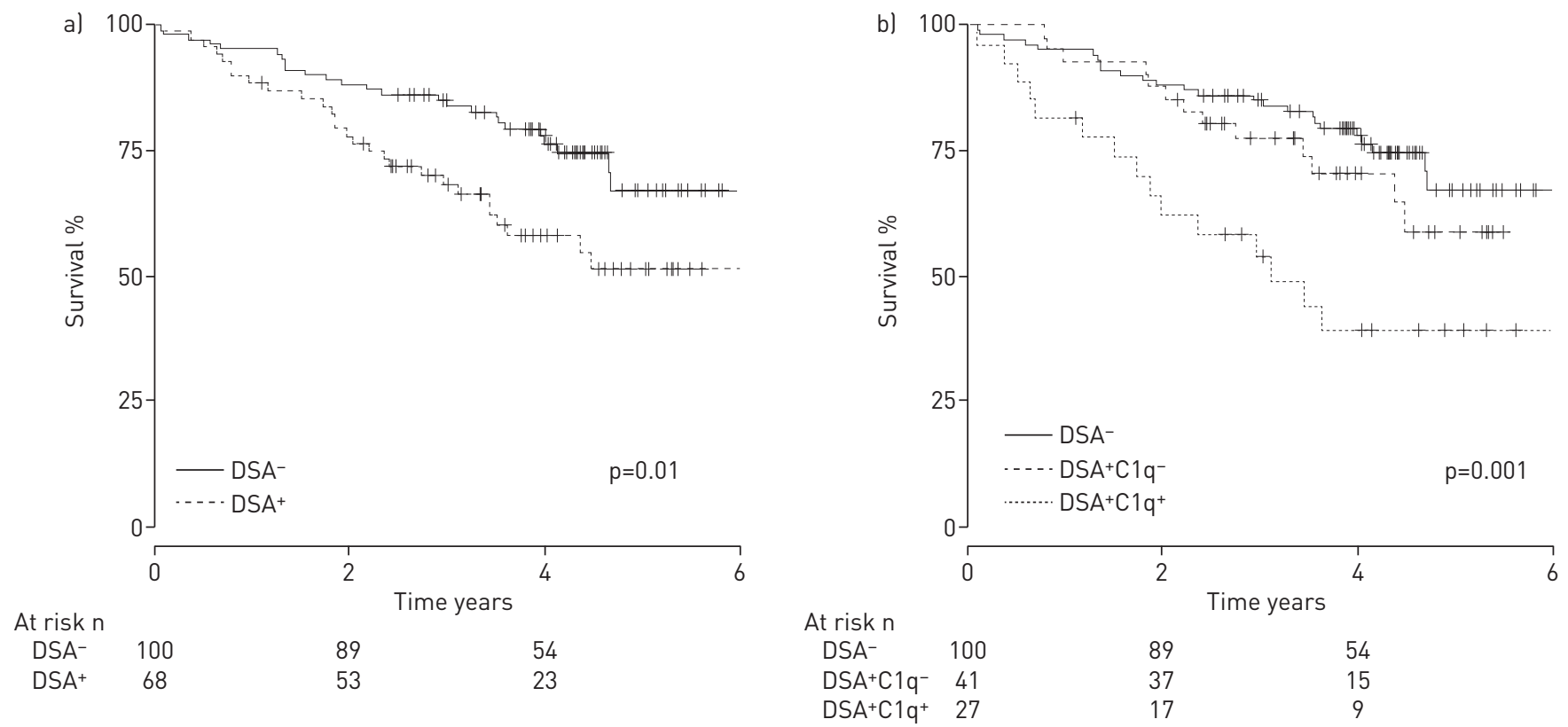

FIGURE 3 Kaplan-Meier curves for graft survival according to donor-specific anti-human leukocyte antigen antibody (DSA) status after transplantation. Probability of graft survival a) by presence or absence of DSA detected using historical Luminex single-antigen flow bead assay; b) by presence or absence of DSAs and their C1q-binding capacity. Survival curves are marked by a vertical tick at each censoring time 
TABLE 2 Clinical, functional, histological and immunological factors associated with chronic lung allograft dysfunction (CLAD) onset and graft survival after lung transplantation

CLAD onset

\section{Univariate analysis}

Recipient age (by 5-unit increase)

Recipient sex (male versus female)

Sex mismatch

Lung tranplant indication

Bilateral

Unilateral

Heart-lung

Initial disease

Emphysema/COPD

Fibrosis

Cystic fibrosis

$\mathrm{PAH}$

Other

CMV mismatch (yes versus no)

Ischaemia time per 60-unit increase

Primary graft dysfunction grade 3 (yes versus no)

Per-operative ECMO or cardiopulmonary bypass (yes versus no)

HLA-A MM

1 HLA-A MM versus 0 HLA-A MM

2 HLA-A MM versus 0 HLA-A MM

HLA-B MM

HLA-DR MM

1 HLA-DR MM versus 0 HLA-DR MM

2 HLA-DR MM versus 0 HLA-DR MM

HLA-DQ MM

1 HLA-DR MM versus 0 HLA-DR MM

2 HLA-DR MM versus 0 HLA-DR MM

Preformed DSA (yes versus nol

Induction therapy

Thymoglobulin (yes versus no)

IL-2 antagonist (yes versus no)

Acute rejection score (by 1-point increase)

DSA after lung transplant (yes versus no)

DSA

$r$-DSA

C1q-binding DSA (yes versus no)

C1q-binding DSA

C1q-binding r-DSA

Groups of patients

No DSA

Non-C1q-binding DSA

C1q-binding DSA

No r-DSA

Non-C1q-binding r-DSA

C1q-binding r-DSA

\section{Multivariate analysis}

No DSA

Non-C1q-binding DSA

C1q-binding DSA

No r-DSA

Non-C1q-binding r-DSA

C1q-binding $r$-DSA
$0.91(0.81-1.01)$

$1.43(0.87-2.34)$

$1.73(091-3.31)$

$$
1.00
$$

$1.11(0.47-2.64)$

$1.31(1.31-1.31)$

1.00
$3.62(1.75-7.48)$
$2.48(1.4-4.41)$
$2.44(1.31-4.56)$
$1.21(0.65-2.26)$
$1.69(0.89-3.18)$
$0.93(0.78-1.11)$
$1.02(0.6-1.75)$
$1.75(1.03-2.98)$
$1.03(0.24-4.37)$
$1.17(0.29-4.75)$
$0.87(0.52-1.47)$
$0.84(0.11-6.32)$
$1.39(0.19-10.08)$
$3.03(0.93-9.88)$
$3.22(1.04-10.04)$
$0.81(0.42-1.57)$

$1.55(0.52-4.62)$

$1.41(0.73-2.7)$

$1.06(0.92-1.23)$

$1.92(1.2-3.07)$

$2.42(1.5-3.91)$

$2.58(1.51-4.41)$

$4.44(2.19-9.01)$

1.00

$1.45(0.82-2.54)$

$2.91(2.07-4.1)$

1.00

$1.99(1.17-3.38)$

5.65 (4-7.98)

$$
1.00
$$

$1.39(0.76-2.56)$

$2.60(1.27-5.32)$ 1.00

$1.89(1.04-3.41)$

8.35 (3.44-20.28)

p-value

Graft failure

p-value

0.07

0.15

0.09

0.75

75

$53(0.87-2.7)$

1.76 (0.85-3.63)

1.00

$0.78(0.3-2.03)$

0.96

0.003

1.00

$2.08(0.94-4.63)$

$1.69(0.95-3.00)$

1.64 (0.87-3.08)

$0.45(0.23-0.86)$

$1.64(0.79-3.4)$

0.85 (0.69-1.05)

1.07 (0.57-2.01)

$1.22(0.64-2.3)$

$1.0(0.23-4.37)$

0.99 (0.24-4.07)

$1.15(0.6-2.2)$

0.60
0.12

0.07

$2.37(0.55-10.14)$

$2.89(0.72-11.63)$

0.52

0.28 (0.1-0.8)

0.71

$1.75(0.49-6.22)$

$1.38(0.62-3.07)$

0.43

1.07 (0.91-1.25)

0.45

0.006

$<0.00001$

$1.82(1.06-3.15)$

2.28 (1.31-3.98)

0.03

0.001

$<0.00001$

$2.41(1.3-4.43)$

$3.68(1.8-7.52)$

0.009

0.003

1.00

$1.36(0.69-2.67)$

2.65 (1.77-3.95)

1.00

$1.69(0.89-3.22)$

4.36 (3.94-6.48)

1.00

$1.55(0.75-3.21)$

$2.98(1.33-6.66)$ 1.00

$1.82(0.88-3.77)$

6.05 (2.50-14.69)

0.68

0.14

0.12

0.87

0.19

0.11

0.84

0.55

0.99

0.68

0.34

0.26

0.006

0.68

0.004

0.002

0.02

0.002

0.23

0.008

0.11

$<0.0001$

$<0.0001$

Data are presented as hazard ratios $(95 \% \mathrm{Cls})$, unless otherwise stated. COPD: chronic obstructive pulmonary disease; PAH: pulmonary arterial hypertension; CMV: cytomegalovirus; ECMO: extracorporeal membrane oxygenation; HLA: human leukocyte antigen; MM: mismatch; DSA: donor-specific antibody; IL: interleukin; r-DSA: reinterpreted EDTA-single-antigen flow bead DSA. 
the presence of donor-specific anti-HLA antibodies and C1q-binding donor-specific anti-HLA antibodies (table 2). On multivariable analysis, we observed that 1) both C1q-binding DSA and C1-binding r-DSA detection were independent predictors of both CLAD onset (HR 2.60, 95\% CI 1.27-5.32; $\mathrm{p}=0.009$ and HR 8.35, 95\% CI 3.44-20.28; $\mathrm{p}<0.0001$, respectively) and graft loss (HR 2.98, 95\% CI 1.33-6.66; $\mathrm{p}=0.008$ and HR 6.05, 95\% CI 2.50-14.69; $\mathrm{p}<0.0001$, respectively); while 2) only detection of non-complement binding r-DSA was an independent predictor of CLAD (HR 1.89, 95\% CI 1.04-3.41; p=0.03 (table 2).

Complement-binding DSA remained independently associated with risk of CLAD development after adjustment for MFI value of DSAs (HR 2.85, 95\% CI 1.09-7.46; p=0.03). Kaplan-Meier survival curves for freedom from CLAD and graft survival stratified by MFI value of DSA in patients with C1q-binding DSA were similar, regardless of whether the mean fluorescence intensity was low $(<2000$ arbitrary units) or high ( $\geqslant 2000$ arbitrary units; $\mathrm{p}=0.92$ and 0.44 , respectively) (figure $4 \mathrm{a}$ and $\mathrm{b}$, respectively). Lastly, we compared patients with and those without preformed DSA, and found no significant difference in freedom from CLAD (log-rank, $\mathrm{p}=0.94$ ) and survival (log-rank, $\mathrm{p}=0.44)$ between the two groups.

\section{Sensitivity analysis}

In the sensitivity analysis, we assessed the robustness of the results by investigating associations separately in each study centre. In each of the three transplantation centres, patients with complement-binding DSA had the highest incidence of CLAD and the lowest rate of graft survival (all $\mathrm{p}<0.05$ ) (online supplementary figure S4).

\section{Severity and CLAD phenotype by type of DSA detection}

At last follow-up, the frequency of severe CLAD was higher with patients with complement-binding DSA $(\mathrm{n}=14,52 \%)$, as compared to patients without DSA $(\mathrm{n}=17,17 \% ; \mathrm{p}=0.0006)$ or non-complement-binding DSA patients $(n=10,24 \% ; p=0.03)$ (online supplementary figure S5). The relative proportions of BOS3 phenotype and severe RAS phenotype at last follow-up were similar among groups $(n=10$ BOS 3 and $n=4$ severe RAS patients with $\mathrm{Clq}^{+} \mathrm{DSA}$, as compared to $\mathrm{n}=10$ BOS3 and $\mathrm{n}=7$ severe RAS patients without DSA and $n=6$ BOS3 and $n=4$ severe RAS patients with non-C1q-binding DSA; $p=0.74$, Chi-squared test).

\section{MFI value of DSA detected by standard SAFB, rEDTA-SAFB and C1q-assay}

The mean \pm SEM MFI value was higher for SAFB-detected DSAs with than without corresponding C1q-binding (4043 $\pm 827.4, \mathrm{n}=27$ versus $2270 \pm 342.1, \mathrm{n}=41 ; \mathrm{p}=0.02$ ) (online supplementary figure S6), and a similar difference was observed for r-DSAs (online supplementary figure S7).

The mean \pm SEM MFI value for all DSAs detected in C1q assay was $4102 \pm 795$. The mean \pm SEM MFI value of DSAs in $\mathrm{C} 1 \mathrm{q}$ assay was similar in $\mathrm{DSA}^{+} \mathrm{Clq}^{+}$patients and $\mathrm{rDSA}^{+} \mathrm{Clq}^{+}$patients $(4671 \pm 1071, \mathrm{n}=27$ versus 2705 $\pm 707, \mathrm{n}=11 ; \mathrm{p}=0.2684$ ) (online supplementary figure $\mathrm{S} 8$ ).

\section{Discussion}

Our study shows that the presence of C1q-binding DSAs detected in the first year after lung transplantation is an independent predictor of CLAD development and graft failure at 1- and 3-years post-detection. The $\mathrm{Clq}$ assay significantly improved the individual risk stratification, with a nearly three-fold increased risk of both CLAD occurrence and graft loss for patients with detected complement-binding DSAs, who had the highest immunological risk as compared to patients without DSAs or with non-complement-fixing DSA. Of note, most of complement-binding DSAs were class II
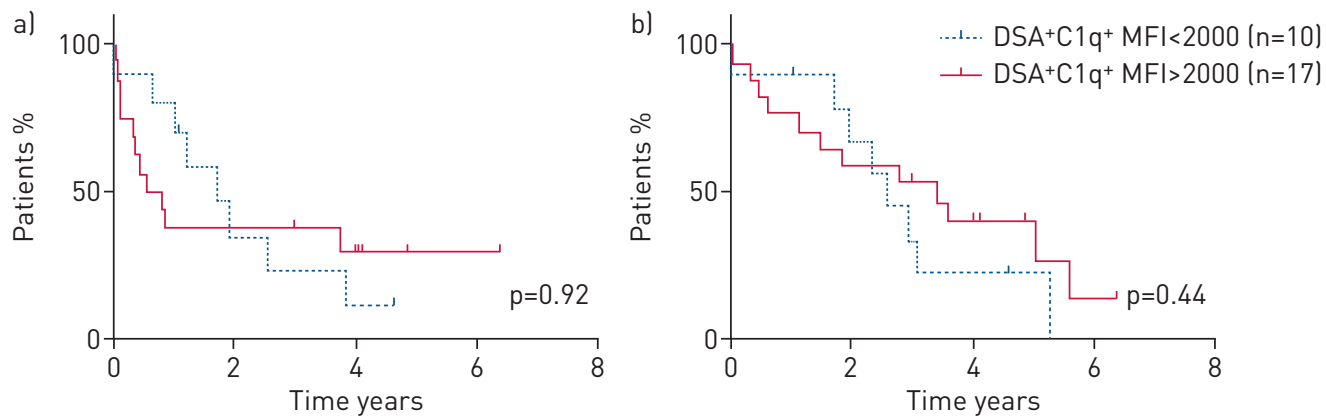

FIGURE 4 Kaplan-Meier curves for freedom from chronic allograft dysfunction (CLAD), stratified by mean fluorescence intensity (MFI) value of C1q-binding donor-specific anti-human leukocyte antigen antibody (DSA) detected using classical Luminex single-antigen flow bead assay (MFI >2000 or <2000) after lung transplantation. Probability of a) freedom from CLAD and b) graft survival by MFI value of C1q-DSAs 
anti-HLA antibodies with DQ specificity, which suggests the crucial role of the locus DQ antigens in the antibody-mediated rejection events linked to complement injury.

Our findings are consistent with previous studies showing a strong association between detection of preformed and/or de novo DSAs and increased risk of graft failure [3-10]. The most convincing demonstration of the immunological risk for graft failure linked to DSA antibodies have been allowed with the recent use of high-sensitive SAFB Luminex technology in the early 2000s $[6,8,9,31,34]$, which revealed a much higher proportion of immunised patients than previously found with the older, less sensitive techniques $[3,35,36]$. In our study, we further extended these results, and showed that as compared with the traditional approach based on only the presence of DSAs after lung transplantation as a risk factor of graft loss, the $\mathrm{C1q}$ assay we used, which integrates the capacity of DSAs to bind complement, identified an additional group of patients with increased risk of CLAD onset and graft loss. Within this group, patients with CLAD had the higher proportion of severe disease as compared with other patients, without a predominance of a specific phenotype (i.e. BOS versus RAS). Furthermore, we looked at a subgroup of 27 patients with available systematic transbronchial biopsies including C4d staining analysis, which were performed close to the date of $\mathrm{Clq}$ sampling (within 1 month before or after). Among them, none had C4d staining $>50 \%$ considered as significant according to recommendations of ISHLT [19]. They included 17 patients without DSA, seven with $\mathrm{Clq}^{-}$DSAs and three with $\mathrm{Clq}^{+}$DSAs. It suggests that $\mathrm{C} 4 \mathrm{~d}$ staining does not seem to correlate to $\mathrm{C}_{1} \mathrm{q}^{+} \mathrm{DSAs}$ detection in stable lung transplantation recipients, but no conclusion can be drawn due to the small number of subjects.

Our results replicate those of a large-population kidney-recipient study by Loupy et al. [37] performed with the collaboration of the same HLA laboratory, upon which an amended design of our study was based. In this cohort of 1016 kidney transplantation recipients, the presence of C1q-binding DSA antibodies detected in the first year after transplantation was also found to be an independent predictor of kidney-allograft loss $>5$ years after transplantation, and associated with the poorest outcome compared with presence of non-C1q-binding DSAs and no DSAs. We found similar results on screening DSAs using the same protocol as for this kidney-transplantation study in Saint-Louis hospital [37]. Concerning the specific risk of non-complement-fixing antibodies, patients with non-C1-binding r-DSA were associated with an increased risk of CLAD/graft failure onset as compared to the group without r-DSA, but this difference was not or only marginally observed when comparing non-C1q-fixing DSA patients and those without DSA. Hence, the lack of complement-fixing property of DSAs or r-DSAs did not exclude their deleterious role in some patients, but the pathogenic non-complement-mediated pathways, such as potential direct epithelial injury [38], or Fc-mediated involvement of macrophages, natural killer or $\gamma \delta$ T-cells [39] remain to be defined in lung transplantation.

During the first year after transplantation, $40 \%$ of our population was considered immunised, which agrees with frequency of $26-61 \%$ found in other lung transplantation recipients screened by SAFB Luminex assay during follow-up $[8,9,14,31]$. We found male sex, HLA DQ- and DR-mismatches and preformed DSAs associated with presence of DSAs within the first 6-12 months post-transplantation. Because females are more likely than males to be sensitised due to pregnancy, the increased proportion of males with DSA we found seems to be inconsistent. Although we have no clear explanation, but the predominance of DSAs in male patients has been reported in lung [9] and kidney transplantation [40]. HLA mismatches at DR or DQ loci have been found to be associated with the development of DSAs after lung transplantation in recent studies at the DQ [9] or DR locus [7], but not in others using the same SAFB Luminex assay [8, 31]. A parallel striking finding in our cohort was the large majority of DQ-specific DSAs detected with both SAFB Luminex and C1q assay, which was also observed in a recent lung transplantation study [9] and kidney transplantation studies using only SAFB Luminex assay [41-43]. Finally, we observed an increased frequency of preformed DSAs in both patients with $\mathrm{C}_{1} \mathrm{q}^{+} \mathrm{DSAs}$ and $\mathrm{C} 1 \mathrm{q}^{-} \mathrm{DSAs}$. This agrees with previous findings from some studies in which preformed DSAs were identified as a risk factor for DSA development after lung transplantation [7, 44], whereas it was not found in other studies [9].

We performed our main analysis of C1q-assay on the historical sera screened from SAFB results which had been used in clinical practice, but we also retested the selected sera with an EDTA-modified SAFB assay which came along with redefinition of DSAs (r-DSA), whenever relevant (i.e. when an antibody reacted with the allele not expressed by the donor antigen) [45]. The r-DSA definition decreased the number of DSA-positive patients and of $\mathrm{DSA}^{+} \mathrm{Clq}$ patients, as compared with the historical DSA definition, but the high prevalence of DQ persisted. For both analyses of DSAs and r-DSAs, MFI values were higher in those with than without corresponding complement-fixing, which agrees with data showing that high MFI values as the most important factor predicting complement activation [46]. Importantly, some patients had MFI values of C1q-binding DSAs between 500 and 2000, so low MFI values does not preclude complement-fixing properties. In addition, the $\mathrm{C} 1 \mathrm{q}$ assay does not require activation of the complement cascade or any functional event such as activating complement split products (e.g. C4d). 
Consequently, it should identify DSAs that might be problematic in the future, even if they are not currently activating the complement cascade.

As a clinical point of view, the use of $\mathrm{C} 1 \mathrm{q}$ assay versus standard SAFB assay in the first year after transplantation could help better identifying tolerant patients, to reduce unnecessary pre-emptive desensitisation therapy, and might identify patients with a higher risk of graft failure early, in an attempt to use anti-complement-targeted therapy [47]. Additionally, the potential utility of repeated C1q testing during follow-up remains to be investigated, because antibody class switching is dynamic, with a possible conversion from a complement-fixing to -non-fixing state, and vice versa.

In conclusion, our results suggest that whatever the DSA SAFB screening assay used, the complement-binding ability of DSA remained the best factor associated with increased immunological risk of both CLAD onset and graft failure after lung transplantation. The complement-binding capacity of DSAs was an independent predictor of poor outcome, independent of the DSA MFI value. Hence, as compared with the standard SAFB assay, the new C1q assay may allow for better stratification of the immunologic risk of CLAD and graft failure in lung transplantation recipients.

Author contributions: O. Brugière: acquisition and analysis of data, writing the manuscript. A. Roux, J. Le Pavec, G. Thabut, J-L. Taupin: designing the study, acquisition and analysis of data, writing the manuscript. D. Sroussi, G. Jebrak, G. Dauriat, C. Dupin, V. Bunel, P. Pradère, G. Mourin, A. Cazes, B. Lortat-Jacob, S. Jean-Baptiste, C. Suberbielle: acquisition and analysis of data. H. Mal, Y. Castier, P. Mordant, S. Caillat-Zucman, F. Parquin, C. Gautreau: designing the study and acquisition of data, reviewing the manuscript.

Conflict of interest: H. Mal reports personal fees from Roche, Boehringer and CSL Behring, outside the submitted work.

Support statement: This study was funded by Vaincre la Mucoviscidose (VLM). Funding information for this article has been deposited with the Crossref Funder Registry.

\section{References}

1 Patel R, Terasaki PI. Significance of the positive crossmatch test in kidney transplantation. N Engl J Med 1969; 280: 735-739.

2 Kotloff RM, Thabut G. Lung transplantation. Am J Respir Crit Care Med 2011; 184: 159-171.

3 Palmer SM, Davis RD, Hadjiliadis D, et al. Development of an antibody specific to major histocompatibility antigens detectable by flow cytometry after lung transplant is associated with bronchiolitis obliterans syndrome. Transplantation 2002; 74: 799-804.

4 Hadjiliadis D, Chaparro C, Reinsmoen NL, et al. Pre-transplant panel reactive antibody in lung transplant recipients is associated with significantly worse post-transplant survival in a multicenter study. J Heart Lung Transplant 2005; 24: S249-S254.

5 Angaswamy N, Saini D, Ramachandran S, et al. Development of antibodies to human leukocyte antigen precedes development of antibodies to major histocompatibility class I-related chain A and are significantly associated with development of chronic rejection after human lung transplantation. Hum Immunol 2010; 71: 560-565.

6 Morrell MR, Pilewski JM, Gries CJ, et al. De novo donor-specific HLA antibodies are associated with early and high-grade bronchiolitis obliterans syndrome and death after lung transplantation. J Heart Lung Transplant 2014; 33: $1288-1294$.

7 Ius F, Sommer W, Tudorache I, et al. Early donor-specific antibodies in lung transplantation: risk factors and impact on survival. J Heart Lung Transplant 2014; 33: 1255-1263.

8 Le Pavec J, Suberbielle C, Lamrani L, et al. De-novo donor-specific anti-HLA antibodies 30 days after lung transplantation are associated with a worse outcome. J Heart Lung Transplant 2016; 35: 1067-1077.

9 Tikkanen JM, Singer LG, Kim SJ, et al. De Novo DQ donor-specific antibodies are associated with chronic lung allograft dysfunction after lung transplantation. Am J Respir Crit Care Med 2016; 194: 596-606.

10 Kim M, Townsend KR, Wood IG, et al. Impact of pretransplant anti-HLA antibodies on outcomes in lung transplant candidates. Am J Respir Crit Care Med 2014; 189: 1234-1239.

11 Tinckam KJ, Keshavjee S, Chaparro C, et al. Survival in sensitized lung transplant recipients with perioperative desensitization. Am J Transplant 2015; 15: 417-426.

12 Yusen RD, Edwards LB, Kucheryavaya AY, et al. The registry of the International Society for Heart and Lung Transplantation: thirty-second official adult lung and heart-lung transplantation report - 2015; focus theme: early graft failure. J Heart Lung Transplant 2015; 34: 1264-1277.

13 Lachmann N, Todorova K, Schulze H, et al. Luminex and its applications for solid organ transplantation, hematopoietic stem cell transplantation, and transfusion. Transfus Med Hemother 2013; 40: 182-189.

14 Hachem RR, Yusen RD, Meyers BF, et al. Anti-human leukocyte antigen antibodies and preemptive antibody-directed therapy after lung transplantation. J Heart Lung Transplant 2010; 29: 973-980.

15 Gebel HM, Bray RA. HLA antibody detection with solid phase assays: great expectations or expectations too great? Am J Transplant 2014; 14: 1964-1975.

16 Feucht HE, Felber E, Gokel MJ, et al. Vascular deposition of complement-split products in kidney allografts with cell-mediated rejection. Clin Exp Immunol 1991; 86: 464-470.

17 Collins AB, Schneeberger EE, Pascual MA, et al. Complement activation in acute humoral renal allograft rejection: diagnostic significance of C4d deposits in peritubular capillaries. J Am Soc Nephrol 1999; 10: 2208-2214.

18 Böhmig GA, Exner M, Habicht A, et al. Capillary C4d deposition in kidney allografts: a specific marker of alloantibody-dependent graft injury. J Am Soc Nephrol 2002; 13: 1091-1099.

19 Levine DJ, Glanville AR, Aboyoun C, et al. Antibody-mediated rejection of the lung: a consensus report of the International Society for Heart and Lung Transplantation. J Heart Lung Transplant 2016; 35: 397-406. 
20 Berry GJ, Burke MM, Andersen C, et al. The 2013 International Society for Heart and Lung Transplantation Working Formulation for the standardization of nomenclature in the pathologic diagnosis of antibody-mediated rejection in heart transplantation. J Heart Lung Transplant 2013; 32: 1147-1162.

21 Roux A, Bendib Le Lan I, Holifanjaniaina S, et al. Antibody-mediated rejection in lung transplantation: clinical outcomes and donor-specific antibody characteristics. Am J Transplant 2016; 16: 1216-1228.

22 Racusen LC, Haas M. Antibody-mediated rejection in renal allografts: lessons from pathology. Clin J Am Soc Nephrol 2006; 1: 415-420.

23 Colvin RB. Antibody-mediated renal allograft rejection: diagnosis and pathogenesis. J Am Soc Nephrol 2007; 18: 1046-1056.

24 Chin C, Chen G, Sequeria F, et al. Clinical usefulness of a novel C1q assay to detect immunoglobulin G antibodies capable of fixing complement in sensitized pediatric heart transplant patients. J Heart Lung Transplant 2011; 30: $158-163$.

25 Brugière O, Suberbielle C, Thabut G, et al. Lung transplantation in patients with pretransplantation donor-specific antibodies detected by Luminex assay. Transplantation 2013; 95: 761-765.

26 Brugière $\mathrm{O}$, Thabut G, Krawice-Radanne I, et al. Role of HLA-G as a predictive marker of low risk of chronic rejection in lung transplant recipients: a clinical prospective study. Am J Transplant 2015; 15: 461-471.

27 Brugière $\mathrm{O}$, Thabut $\mathrm{G}$, Mal H, et al. Exhaled NO may predict the decline in lung function in bronchiolitis obliterans syndrome. Eur Respir J 2005; 25: 813-819.

28 Cooper JD, Gaissert HA, Patterson GA, et al. Die Lungenvolumenreduktionschirurgie des fortgeschrittenen Emphysems - Ergebnisse der Washington University, St. Louis. [Lung volume reduction surgery in advanced emphysema - results of the Washington University, St. Louis]. Wien Med Wochenschr 1996; 146: 592-598.

29 Verleden GM, Raghu G, Meyer KC, et al. A new classification system for chronic lung allograft dysfunction. J Heart Lung Transplant 2014; 33: 127-133.

30 Verleden GM, Vos R, Verleden SE, et al. Survival determinants in lung transplant patients with chronic allograft dysfunction. Transplantation 2011; 92: 703-708.

31 Safavi S, Robinson DR, Soresi S, et al. De novo donor HLA-specific antibodies predict development of bronchiolitis obliterans syndrome after lung transplantation. J Heart Lung Transplant 2014; 33: 1273-1281.

32 Grambsch PM, Therneau TM. Proportional hazards tests and diagnostics based on weighted residuals. Biometrika 1994; 81: 551-526.

33 Schemper M, Smith TL. A note on quantifying follow-up in studies of failure time. Control Clin Trials 1996; 17: 343-346.

34 Lobo LJ, Aris RM, Schmitz J, et al. Donor-specific antibodies are associated with antibody-mediated rejection, acute cellular rejection, bronchiolitis obliterans syndrome, and cystic fibrosis after lung transplantation. J Heart Lung Transplant 2013; 32: 70-77.

35 Smith MA, Sundaresan S, Mohanakumar T, et al. Effect of development of antibodies to HLA and cytomegalovirus mismatch on lung transplantation survival and development of bronchiolitis obliterans syndrome. J Thorac Cardiovasc Surg 1998; 116: 812-820.

36 Girnita AL, McCurry KR, Iacono AT, et al. HLA-specific antibodies are associated with high-grade and persistent-recurrent lung allograft acute rejection. J Heart Lung Transplant 2004; 23: 1135-1141.

37 Loupy A, Lefaucheur C, Vernerey D, et al. Complement-binding anti-HLA antibodies and kidney-allograft survival. N Engl J Med 2013; 369: 1215-1226.

38 Jaramillo A, Smith CR, Maruyama T, et al. Anti-HLA class I antibody binding to airway epithelial cells induces production of fibrogenic growth factors and apoptotic cell death: a possible mechanism for bronchiolitis obliterans syndrome. Hum Immunol 2003; 64: 521-529.

39 Bachelet T, Couzi L, Pitard V, et al. Cytomegalovirus-responsive $\gamma \delta \mathrm{T}$ cells: novel effector cells in antibody-mediated kidney allograft microcirculation lesions. J Am Soc Nephrol 2014; 25: 2471-2482.

40 Hirai T, Furusawa M, Omoto K, et al. Analysis of predictive and preventive factors for de novo DSA in kidney transplant recipients. Transplantation 2014; 98: 443-450.

41 Willicombe M, Brookes P, Sergeant R, et al. De novo DQ donor-specific antibodies are associated with a significant risk of antibody-mediated rejection and transplant glomerulopathy. Transplantation 2012; 94: 172-177.

42 Wiebe C, Pochinco D, Blydt-Hansen TD, et al. Class II HLA epitope matching-A strategy to minimize de novo donor-specific antibody development and improve outcomes. Am J Transplant 2013; 13: 3114-3122.

43 Freitas MC, Rebellato LM, Ozawa $\mathrm{M}$, et al. The role of immunoglobulin-G subclasses and $\mathrm{Clq}$ in de novo HLA-DQ donor-specific antibody kidney transplantation outcomes. Transplantation 2013; 95: 1113-1119.

44 Snyder LD, Wang Z, Chen DF, et al. Implications for human leukocyte antigen antibodies after lung transplantation: a 10-year experience in 441 patients. Chest 2013; 144: 226-233.

45 Tambur AR, Rosati J, Roitberg S, et al. Epitope analysis of HLA-DQ antigens: what does the antibody see? Transplantation 2014; 98: 157-166.

46 Tambur AR, Herrera ND, Haarberg KM, et al. Assessing antibody strength: comparison of MFI, C1q, and titer information. Am J Transplant 2015; 15: 2421-2430.

47 Viglietti D, Gosset C, Loupy A, et al. C1 inhibitor in acute antibody-mediated rejection nonresponsive to conventional therapy in kidney transplant recipients: a pilot study. Am J Transplant 2016; 16: 1596-1603. 